\title{
Vers un métamodèle pour analyser les systèmes d'élevage extensifs et leurs interactions avec les territoires
}

Pierre Bommel ${ }^{1,2}$

Alassane $\mathrm{Bah}^{3}$

Michel Etienne ${ }^{4}$

Grégoire Leclerc ${ }^{1}$

Claude Montei ${ }^{5,6}$

Ibra Toure ${ }^{7}$

\section{${ }^{1}$ Cirad UPR GREEN}

Campus international de Baillarguet

TAC-47 / F

34398 Cedex Montpellier

<bommel@cirad.fr>

<gregoire.leclerc@cirad.fr>

${ }^{2}$ Université de Brasília, Faculté de technologie Campus Universitário Darcy Ribeiro

Asa Norte, 70.910-900

Brasília

Brésil

<bommel@cirad.fr>

3 École polytechnique de Dakar

Dakar

Sénégal

<bah@ucad.sn>

${ }^{4}$ Inra

Unité d'écodéveloppement

Avignon

France

<Michel.Etienne@avignon.inra.fr>

${ }^{5}$ Université de Toulouse, INPT-Ensat

UMR 1201 DYNAFOR

F-31326 Castanet Tolosan

France

<monteil@ensat.fr>

${ }^{6}$ Inra

UMR 1201 DYNAFOR

F-31326 Castanet Tolosan

France

<monteil@ensat.fr>

7 Cirad

URP Pastoralisme

ISRA/LNERV

Montpellier

France

<ibra.toure@cirad.fr>

Tirés à part : P. Bommel

\section{Résumé}

Nous proposons un canevas souple et adaptable pour faciliter la conception rapide de modèles permettant d'aborder la durabilité des systèmes d'élevage extensif à l'échelle de territoires. Ce métamodèle est le fruit d'une démarche de conception à partir de systèmes multi-agents (SMA) développés sur sept sites en Amérique latine, en France et au Sénégal. Il est composé de quatre modules: Agent, Collectivité, Espace, Végétationtroupeau. Nous les illustrons par les exemples tirés des modèles élaborés sur les différents sites. Le rôle de cet outil est discuté afin d'en montrer les avantages et les limites.

Mots clés : élevage extensif ; métamodèle ; système multi-agents.

Thèmes : productions animales ; ressources naturelles et environnement ; territoire, foncier, politique agricole et alimentaire.

\section{Abstract \\ Towards a metamodel to analyze the dynamics of livestock, resources and territories}

We propose a flexible and adaptable framework to assist the quick design of models dealing with the durability of the extensive livestock systems at the territorial scale. This metamodel results from the collaborative design starting from MAS developed on 7 sites in Latin America, France and Senegal. It is composed of four packages: Agent, Community, Space and Vegetation-Herd. We illustrate these packages with examples extracted from the various models. The role of this tool is discussed in order to show its advantages and its limits.

Key words: extensive husbandry; metamodels; multiagent system.

Subjects: animal productions; natural resources and environment; territory, land use, agricultural and food policy.

$\mathrm{C}$ et article présente les premiers résultats de la recherche d'un modèle fédérateur construit à partir de plusieurs modèles multi-agents développés dans le cadre du projet Trans (Transformations des élevages et dynamiques des espaces) financé par l'Agence nationale de la recherche (ANR). Trans vise à systématiser les connaissances sur les systèmes d'élevage extensifs (SEE) à partir de travaux menés dans des sites contrastés. Certains travaux ont débouché sur le développement de systèmes multi-agents (SMA). L'objectif principal de ces SMA est de rendre compte de la durabilité des SEE en s'appuyant sur une démarche d'accompagnement (ComMod, 2005).

Au-delà des différences notables entre les dispositifs, entre les objectifs des modèles et la façon dont ont été conduites les modélisations, des points communs et des convergences apparaissent. Cette capitalisation sert de base à un cadre général pour analyser et concevoir de nouveaux modèles traitant de la durabilité des SEE.

Après une présentation succincte du métamodèle, nous illustrons ses composants à travers les différentes études de cas. Nous présentons aussi les aspects liés aux dynamiques du modèle général et en expliquons les limitations. 


\section{Chercher des points}

\section{communs au-delà des particularités}

La réalisation de ce métamodèle n'est pas le fruit d'une démarche descendante qui consisterait à concevoir a priori un modèle générique décliné ensuite sur chaque terrain. Des expériences antérieures consistant à produire a priori un tel modèle nous ont forcés à reconnaître les défauts de cette façon de procéder. Au contraire, la conception de ce métamodèle a demandé un travail a posteriori de comparaison, de mise en perspective et de généralisation à partir des modèles développés par des équipes autonomes dans diverses régions du monde: en France - modèles " Lubéron " (Lasseur et al., 2010), SMASH ${ }^{1}$ (Monteil et al., 2008) et "Larzac " (Etienne et al., 2010) -, au Sénégal - modèle AIDA, au Ferlo (Bah et al., 2010) - et en Amérique latine modèles "Pampa " et "TransAmazon " (Bommel et al., 2010).

Souvent, il a fallu adapter les modèles originaux pour saisir leurs points communs. Il ne s'agissait ni d'une simple agrégation de concepts ni de garder le "plus petit dénominateur commun ", mais plutôt d'identifier et de regrouper les questions soulevées par chaque modèle et d'extraire les similarités. Ainsi, même si elles étaient nommées différemment, des entités communes ont été identifiées et leurs caractéristiques précisées, ainsi que les relations qui les lient. Il s'agit d'un véritable travail de conception à plusieurs qui implique, de la part de chacun, d'écouter les critiques à l'égard de son propre modèle, d'accepter d'autres façons de le représenter et d'apprécier la manière dont les autres ont abordé tel ou tel aspect. Ce métamodèle est donc le fruit d'une longue négociation.

\section{Modèle générique, général ou métamodèle?}

Ce travail vise à proposer un canevas souple et adaptable pour faciliter la conception de modèles traitant des SEE à l'échelle de territoires. L'objectif n'est pas de trouver un modèle générique à l'ensemble des terrains, mais de proposer un cadre général d'analyse, à partir

\footnotetext{
${ }^{1}$ SMASH : Spatialised Multi-Agent System for
} ASH colonisation of landscape. duquel seraient dérivés plus facilement des modèles adaptés à chaque contexte. Pour faciliter le développement de futurs SMA, ce patron d'analyse est implémenté dans Cormas (Bousquet et al., 1998) qui a constitué la plateforme de simulation privilégiée du projet.

La dénomination de ce modèle a posé problème : fallait-il l'appeler "modèle générique " ou "général " ou " métamodèle "? Le terme " générique " impliquerait qu'on ait réussi à concevoir un modèle commun à tous les SEE. La généricité s'avère souvent un des objectifs de la modélisation : plutôt que proposer un modèle descriptif par contexte (de façon $a d$ hoc), un modèle générique est capable de représenter chaque configuration particulière. Les éléments et les caractéristiques qu'il contient sont communs à chaque terrain et s'avèrent pertinents pour représenter tout SEE. En d'autres termes, il a acquis une haute valeur informative (Bommel, 2009), à la fois abstrait et suffisamment pertinent pour illustrer n'importe quel SEE.

Dans le domaine de la modélisation, le terme " général " véhicule également l'idée que le modèle proposé convient à tout système et qu'il est universel. Si nous étions parvenus à ce stade, nous aurions contribué à améliorer fortement la connaissance des systèmes d'élevage, mais il nous semble encore vain d'espérer trouver un tel modèle capable de décrire à la fois le contexte de l'élevage sahélien et amazonien, par exemple, sans tomber dans les platitudes. Le préfixe "méta- " exprime un changement de niveau. Un métamodèle serait donc un modèle de modèles (Goldspink, 2000) : un outil de description de modèles, à l'instar d'une métadonnée, laquelle sert à définir ou à décrire d'autres données. Mais ce saut sémantique peut être plus limité. En génie logiciel, un métamodèle est un outil pour concevoir des modèles sur un thème prédéfini. Servant à exprimer les concepts communs à un domaine, il en délimite le cadre et les contraintes et facilite le développement d'applications. Ainsi, à partir d'une métaphore sociale, Ferber et Gutknecht (1998) considèrent un SMA comme une organisation particulière dans laquelle tout agent joue un rôle dans chaque groupe auquel il appartient. Ce métamodèle "Agent-groupe-rôle" (AGR) peut s'appliquer à un grand nombre de types d'organisation. Il constitue un schéma pratique pour caractériser un système. L'implémentation d'AGR dans la plateforme MadKit facilite la création de SMA obéissant à ce schéma et facilite leur exécution.
Même si nous ne sommes pas au point de produire un métamodèle dans une forme qui exprime un saut sémantique, ces définitions nous poussent à préférer ce terme, plutôt que celui de "modèle générique ", car, en l'état, il facilite l'analyse et la conception de modèles sans prétendre fournir un modèle général à tout SEE.

\section{Principales questions étudiées}

La finalité générale des modèles SMA développés dans Trans est l'appui aux acteurs locaux dans la recherche de voies de développement durable de l'élevage extensif. La problématique commune consiste à étudier l'influence des facteurs sociaux et environnementaux sur la durabilité de l'élevage et, réciproquement, la façon dont l'élevage pèse sur la durabilité des écosystèmes. Bien sûr, ces problématiques générales ont été déclinées sur chaque terrain. En fonction du contexte, les équipes en place ont élaboré leur modèle sur un objectif plus spécifique (tableau 1). Ainsi, chaque processus de modélisation est singulier et conduit à la définition d'objectifs différents. Or, c'est cette définition qui fixe le cadre d'un modèle, en restreint les dimensions et permet de comprendre pourquoi certains aspects du système étudié ont été ignorés (Grimm et Railsback, 2005). Même si un objectif commun a été partagé par les différents collectifs de chercheurs, sa déclinaison sur les terrains a conduit à des questions différentes. Certains ont mis l'accent sur les aspects fonciers dans un contexte de changement global et de libéralisation des marchés, d'autres sur la déprise des parcours et ses conséquences sur l'envahissement des prairies naturelles, ou, à l'inverse, sur l'impact de l'élevage sur l'écosystème naturel. Toujours est-il que les questions abordées par chaque modélisation ne sont pas identiques. Appliquées à des contextes variés, elles conduisent à des modèles forcément différents. L'enjeu consiste donc à harmoniser les différents points de vue contextuels sur les SEE.

\section{Présentation du métamodèle}

Même si d'autres outils ont été utilisés pour la conception des modèles, le langage de description utilisé par les modélisateurs 


\section{Tableau 1. Objectifs spécifiques des systèmes multi-agents (SMA) de Trans (Transformations des élevages et dynamiques des espaces).}

Table 1. Specific purposes of MAS models of TRANS project.

\begin{tabular}{|c|c|c|}
\hline Région & Site & Objectifs des modèles \\
\hline \multirow[t]{2}{*}{$\begin{array}{l}\text { Amérique } \\
\text { du Sud }\end{array}$} & Amazonie & $\begin{array}{l}\text { Comment les petits colons des fronts pionniers utilisent-ils la terre ? } \\
\text { Quels sont les points clés qui influencent les dynamiques d'utilisation du sol ? } \\
\text { Les questions ont évolué avec le modèle: } \\
\text { - quelles gestions seraient en mesure de concurrencer le système abattis-brûlis-pâturage ? } \\
\text { - quelles sont les mesures et les politiques appropriées pour parvenir à ces fins (mesures incitatives } \\
\text { ou répressives, politiques d'accès au crédit, programmes d'éducation...)? }\end{array}$ \\
\hline & Pampa & $\begin{array}{l}\text { Élaborer deux outils participatifs de monitoring prenant en compte l'évolution des mentalités } \\
\text { "Arapey " (Uruguay): comment la fonction de production varie selon les stratégies d'investissement } \\
\text { et de chargement des parcelles? } \\
\text { "DinamicaParcelaria " (Argentine) : comprendre les relations entre l'agriculture traditionnelle et les } \\
\text { grandes entreprises agricoles capitalistiques (fonds de pension), et anticiper les changements liés à la } \\
\text { concentration de la production agricole }\end{array}$ \\
\hline \multirow[t]{3}{*}{ France } & Larzac & $\begin{array}{l}\text { Gestion sylvo-pastorale : comment limiter l'envahissement par le pin de zones de prairies naturelles } \\
\text { et de parcours, dans des espaces en déprise? }\end{array}$ \\
\hline & Lubéron & $\begin{array}{l}\text { Établir un diagnostic prospectif sur l'élevage ovin et la sylviculture } \\
\text { Comment maintenir le parc naturel régional du Lubéron par les activités d'élevage et sylvicoles? }\end{array}$ \\
\hline & Pyrénées & $\begin{array}{l}\text { Comprendre les causes du boisement par le frêne en relation avec la transformation des activités } \\
\text { agricoles } \\
\text { Évaluer les conséquences de ce processus pour le développement des territoires de montagne } \\
\text { pyrénéenne pour différents scénarios de changement de l'utilisation des terres }\end{array}$ \\
\hline Sénégal & Ferlo & $\begin{array}{l}\text { Étudier les processus d'affectation des terres et leurs impacts sur les différents systèmes de } \\
\text { production (principalement l'élevage) et sur le paysage autour du lac de Guiers }\end{array}$ \\
\hline
\end{tabular}

pour le métamodèle est l'UML (Unified Modeling Language) (OMG, 2003), qui s'avère un outil de dialogue performant entre modélisateurs. Plusieurs versions furent débattues pour aboutir au diagramme général présenté dans la figure 1.

\section{Une structure partagée}

La structure de ce canevas est subdivisée en quatre modules :

- le module "Espace " décrit la manière dont est structuré le territoire. L'ensemble des modèles décrivent des parcelles élémentaires qui sont souvent agrégées en entités de plus haut niveau de type propriété, parcelle cadastrale, parcelle de gestion, etc.

- le module "Végétation-troupeau " décrit les entités de type végétal ou animal qui occupent l'espace ;

- le module "Agent " est le plus compliqué. Un agent est une famille associée à un ou des métiers. Lorsqu'il gère une exploitation, il planifie des " activités agricoles " (sous-module) en fonction de sa stratégie de production du moment ;

- le module "Collectivité " regroupe les entités collectives telles qu'une administration ou un collectif de producteurs, ainsi que le marché.
Chacune de ces parties est décrite en s'appuyant sur les différents SMA développés dans TRANS.

\section{Illustration du module " Espace " à travers les modèles " DinámicasParcelas " et SMASH (Pyrénées)}

Comme pour tout modèle spatialement discrétisé, le choix de la granularité minimale est délicat. Le module "Espace " (figure 1, cadre bleu) n'impose pas de dimension spatiale élémentaire. L'attribut " surface " de " parcelle élémentaire " peut être renseigné différemment pour chaque modèle et peut prendre des valeurs différentes pour chaque entité spatiale élémentaire (ESE ou pixel) si on choisit de générer une carte à partir de données vectorielles.

L'intérêt de ce module est de permettre qu'une ESE appartienne à deux entités de niveau supérieur. Ce niveau d'agrégation permet un découpage de l'espace selon deux aspects :

- le foncier selon un découpage en parcelles cadastrales ;

- le territoire d'exploitation selon un découpage en parcelles de gestion.
Ce principe est illustré de façon simplifiée par le modèle "DinamicaParcelaria " (figure 2). Deux associations relient une parcelle aux agents : l'une décrit la relation de possession (propiétaire $\leftrightarrow$ propriété) et l'autre la relation de gestion (gérant $\leftrightarrow$ exploitation). Ainsi, seuls les exploitants familiaux peuvent acheter ou vendre des terres et décident d'en louer une partie et d'en garder une autre pour la cultiver. L'entreprise (fonds de pension) ne choisit pas la stratégie d'achat: elle ne cherche qu'à louer pour produire. Ainsi, tout agent qui gère de la terre peut produire du soja ou de l'élevage selon ses préférences. L'entreprise choisit systématiquement d'implanter du soja pour lequel elle possède une technologie plus avancée qui lui procure une productivité de $30 \%$ supérieure.

On retrouve cette double relation à la terre dans le modèle SMASH, où le territoire de chaque exploitation croise deux types d'entités spatiales associées respectivement à deux types de dynamiques :

- les parcelles de gestion, qui rendent compte de la gestion technique (fauche, mise au pâturage) ;

- les parcelles cadastrales, qui relèvent des questions de transmission et d'évolution du patrimoine. 


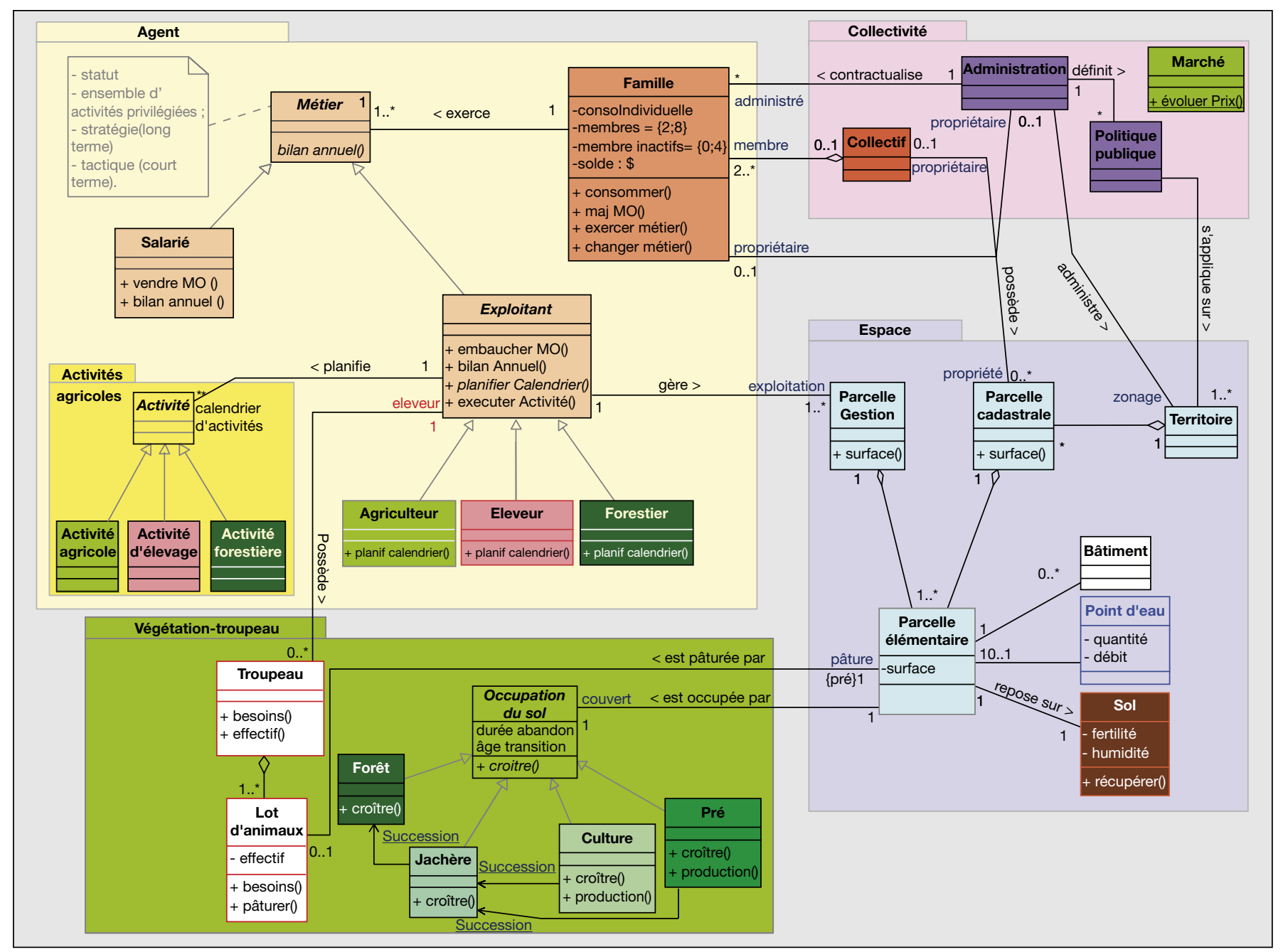

Figure 1. Diagramme de classe général du métamodèle.

Figure 1. Main class diagram of the metamodel. MO : Main-d'œuvre.

Chaque parcelle de base (ESE) appartient donc à deux entités de niveau supérieur qui forment deux couches spatiales distinctes.

\section{Illustration de la partie "Végétation-troupeau " à travers le modèle SMASH}

Distinguer l'occupation de sol (Land Cover) de son utilisation (Land Use) fut l'objet de débats sur la sémantique associée à ces deux notions.

L'occupation du sol, terme retenu pour le métamodèle, traduit le couvert végétal d'une portion d'espace à un instant donné (figure 1, cadre vert). Son évolution obéit à l'action de deux dynamiques conjointes, une naturelle - succession - et une liée aux activités humaines (figure 4). L'implantation d'une prairie résulte du travail de l'éleveur et de la croissance naturelle de l'herbe. Elle demeure dans cet état tant qu'elle est entretenue par la fauche ou le pâturage. En cas d' "abandon " d'usage, elle est colonisée par des accrus et se transforme progressivement en jachère "succession „ puis en forêt. C'est lorsque le Land Cover traduit la conséquence d'actions anthropiques ou de leur absence d'action qu'il y a confusion avec le Land Use. Ainsi, on peut associer deux modalités d'usage à la prairie: "pré de fauche" sur lequel l'exploitant effectue une fauche par an ; "pacage" sur lequel il fait pâturer son troupeau. Ces usages associent une suite d'opérations, chacune devant être réalisée dans une période prédéterminée (itinéraire technique prévisionnel), comme cela est fait dans SMASH, TransAmazon et AIDA.

\section{Illustration du module " Agent " à travers le modèle " TransAmazon "}

Ce module (figure 1, cadre jaune) présente la structure de l'entité "Agent ", qui correspond à la famille de l'exploitant. Aucun modèle n'en distingue les membres. Pour tous les terrains étudiés, l'élevage s'avère une activité qui engage toute la famille. Certes, on observe des activités professionnelles en dehors de l'exploitation (vente de main d'œuvre, travail partiel des femmes hors de la ferme...), mais 


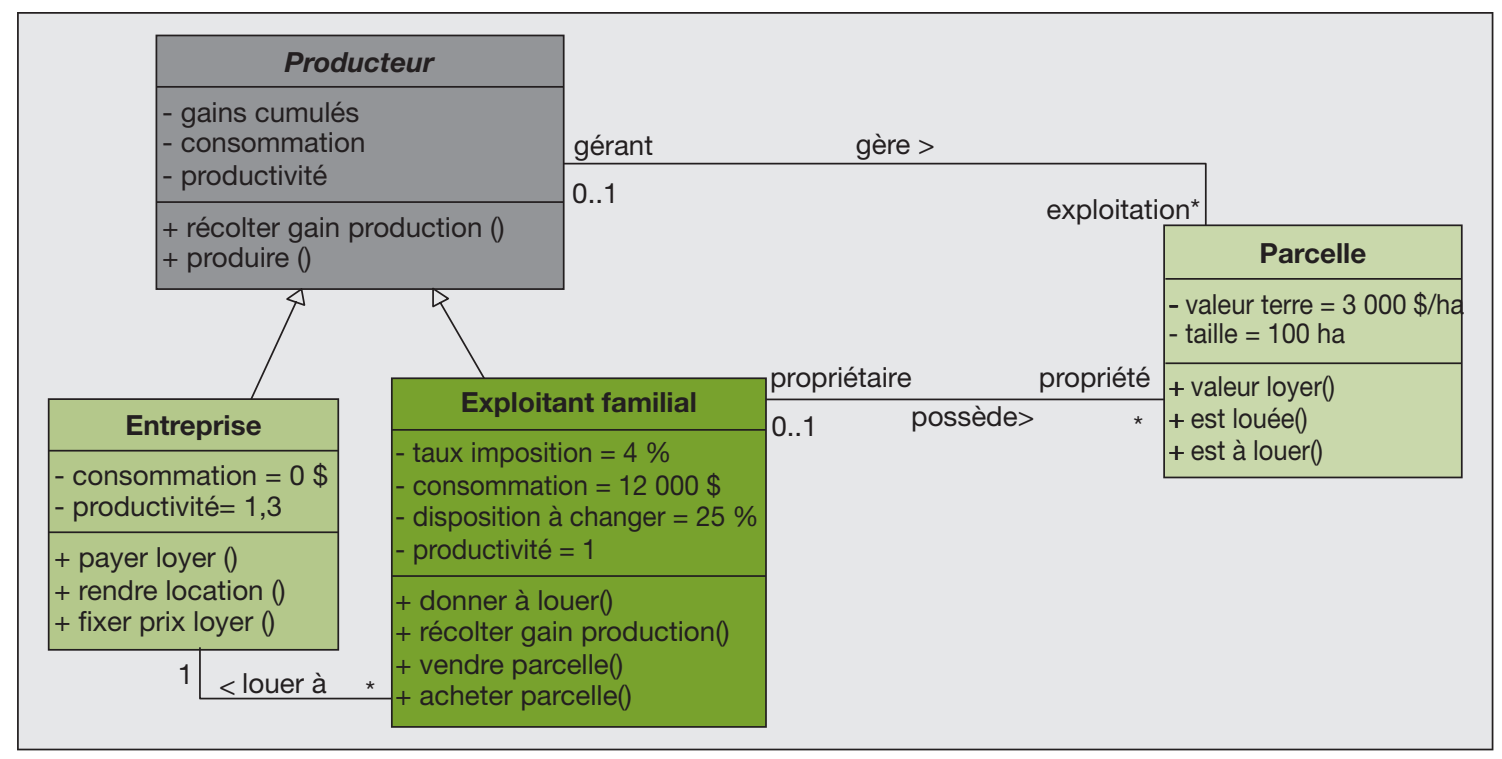

Figure 2. Diagramme de classe de « DinamicaParcelaria » (Pampa).

Figure 2. Class diagram of DinamicaParcelaria (Pampa).

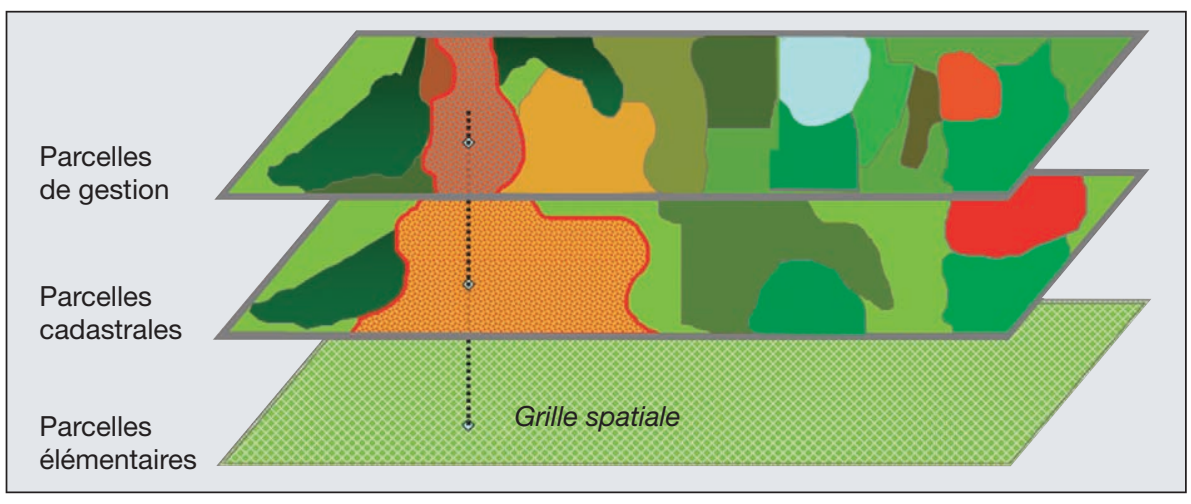

Figure 3. Les trois couches spatiales: les parcelles élémentaires composent les parcelles de gestion et les parcelles cadastrales.

Figure 3. The 3 spatial layers: the spatial elementary plots are the components of the management plots and the cadastral plots.

elles sont souvent marginales en proportion, même si leur revenu aide au maintien économique de la famille. Une famille travaillant complètement en dehors de l'exploitation n'est plus rattachée au SEE que par son lien de propriété.

La deuxième raison de cette nondistinction des membres réside dans l'impossibilité de décrire les activités pour chacun. La complexité devient insurmontable pour peu que l'on veuille traiter de l'évolution individuelle de chacun (son parcours, ses préférences, sa force de travail en fonction de son âge, etc) ou bien des grands événements de la famille (naissance, mariage, maladie, décès, héritage et transmission).
Mis à part, le cas de "Arapey " (Pampa) dans lequel l'éleveur est spécialisé de façon définitive, les agents des modèles peuvent évoluer et changer de comportement au cours du temps. C'est la raison pour laquelle à une famille est associé un métier, qui, selon les cas, peut être vu comme un ensemble d'activités privilégiées ou une stratégie (long terme), voire une tactique (court terme). L'intérêt de séparer l'agent en deux parties est de faciliter la conception en permettant aux modélisateurs de se concentrer sur un seul aspect, sans mélanger des notions rattachées strictement à la famille. De plus, la possibilité de choisir une stratégie agricole et de pouvoir en changer au cours du temps engendre une diversité de parcours familiaux qui procure une richesse de comportements. Ce métier se subdivise en deux types:

- le salarié travaillant hors de l'exploitation ;

- l'exploitant qui gère une exploitation. La figure 5 (TransAmazon) illustre cette séparation de l'agent en deux parties interconnectées. Ici, la stratégie se décline en trois spécialisations : "sans terre ", "éleveur "et " planteur ". Si la première n'est pas issue d'un choix, les deux autres dépendent de décisions prises lors du bilan annuel. L'agriculteur est capable de déclencher un certain nombre d'opérations agricoles (supprimer, implanter, entretenir). L'ordonnancement et la réalisation de ces activités pendant la saison sèche et la saison humide sont spécifiés au niveau des deux sousclasses : "Éleveur " et "Planteur ". En privilégiant les activités liées à la stratégie choisie, l'agriculteur ne délaisse pas pour autant les autres cultures déjà présentes sur son lot. Mais le choix de ces activités agricoles et leur ordonnancement ne sont pas assez explicites sur ce schéma. Il faut présenter des diagrammes d'activités pour comprendre que ces stratégies ne sont pas exclusives.

La solution présentée par le métamodèle est plus claire, car elle montre que l'exploitant connait un certain nombre d'activités agricoles et qu'il peut les planifier pour mettre au point un calendrier des travaux qui privilégie sa stratégie. L'ordonnancement de ces activités est 


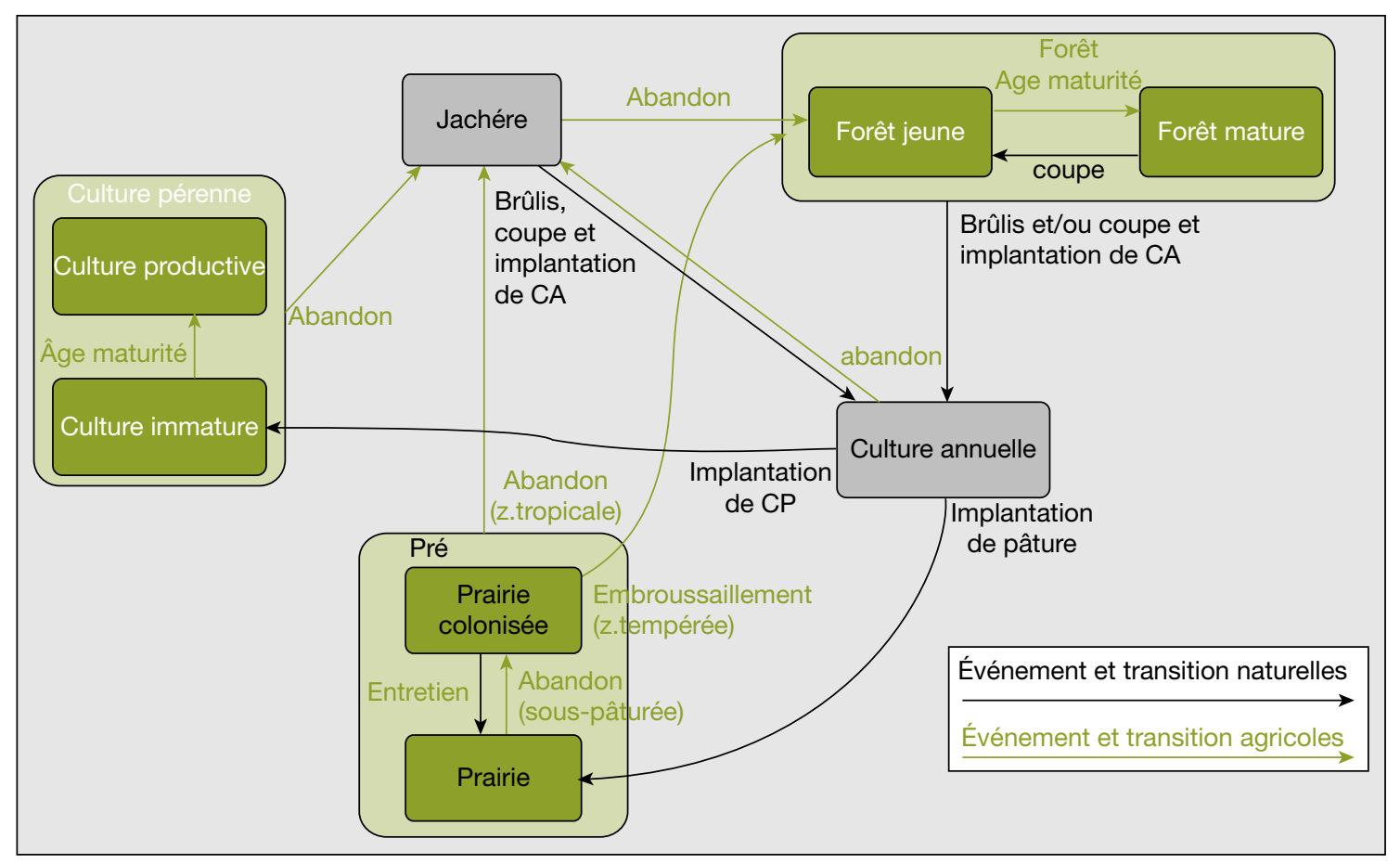

Figure 4. Diagramme d'état-transition de la couverture végétale.

Figure 4. Transition-State diagram of land cover. $\mathrm{CP}$ : Culture pérenne; CA: Culture annuelle.

spécifique à chaque contexte et la mise au point du calendrier est à la charge du concepteur.

\section{Description de la partie "Collectivité "}

Ce module (figure 1, cadre rose) présente les entités collectives qui correspondent souvent à des entités immatérielles : un collectif de producteurs, un réseau d'acteurs, le marché... Dans AIDA, l'affectation des terres résulte d'une décision impliquant le conseil rural, la souspréfecture, les chefs de village, etc.

A l'échelle d'un territoire, le comportement du marché n'est pas lié aux activités marchandes des agents. Dans "Pampa" ou "TransAmazon ", le marché fixe les prix des produits agricoles qui jouent le rôle de variables de forçage : les prix sont imposés de façon externe. Il en va de même pour la politique publique dont les règles sont imposées de façon externe au modèle. Pour certains, le collectif désigne simplement la façon dont les agents se coordonnent et interagissent. D'autres la voient comme une entité à part entière (réification) qui régit les comportements de ses membres (contraintes, règles conventionnelles, etc.). Orléan (2004) définit le groupe social comme une entité auto- nome. Il prend l'exemple de la phrase suivante : "le marché croit que cette devise est sous-évaluée ". Évidemment un marché n'a pas la faculté de croire. Mais il a une existence propre qui dépasse la simple composition d'agents. Un marché ou une collectivité perdure malgré le flux d'agents qui en sort ou y entre. En fonction de la façon dont on perçoit une organisation, on pourra donc la représenter comme la résultante des interactions entre les entités ou, si on lui reconnaît une certaine autonomie, on peut la modéliser comme un agent. Le métamodèle fait le choix de réifier différentes organisations, mais les considère comme des entités périphériques dont les comportements simples sont imposés par le modélisateur pour tester l'influence d'une politique ou d'un marché sur le comportement des agents.

\section{Échelles}

\section{de modélisation spatio-temporelle}

Tous les modèles privilégient la représentation matricielle qui facilite la simulation de processus de diffusion/ contraction tels que la progression d'un front pionnier. Ils montrent une large amplitude d'échelles spatiales (tableau 2). Le découpage de l'espace est déterminé par rapport :

- aux entités de gestion associées aux décisions des agents (l'alqueire en Amazonie, la parcelle de gestion dans les Pyrénées et le Lubéron, le territoire associé à chaque communauté rurale au Ferlo) ;

- aux entités écologiques fonctionnelles associées aux processus biophysiques (peuplement, bassin-versant, zone de dissémination des pins au Larzac);

- aux entités juridiques associées au statut foncier du territoire (cadastre, réglementation définie sur une zone communale, gestion différente selon le statut domanial ou privé).

Ces différents types d'entités peuvent être croisées et donner lieu à des découpages élémentaires plus fins (croisement de parcelles de gestion et de parcelles cadastrales pour le modèle SMASH).

L'étendue spatiale est définie, soit relativement à la question traitée (territoire de la Société civile des terres du Larzac, ou celui du syndicat des communautés rurales au Ferlo), soit par un élément structurant (le travesão en Amazonie), soit par la 


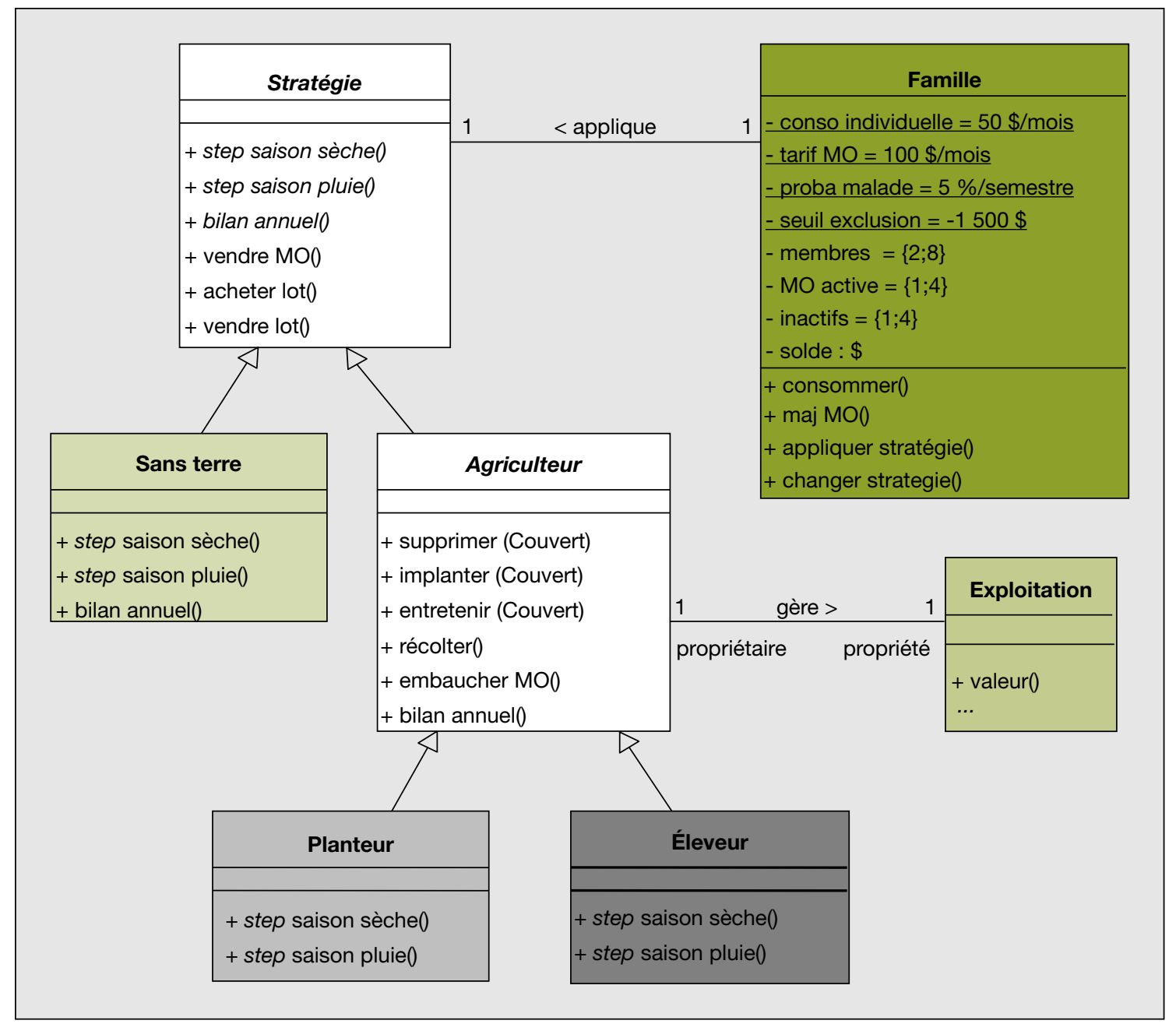

Figure 5. Diagramme de classe des agents de TransAmazon.

Figure 5. Agent class diagram of TransAmazon.

MO : main d'œuvre.

\section{Tableau 2. Les échelles d'espace des modèles.}

Table 2. Spatial scales of the models.

\begin{tabular}{|c|c|c|c|c|}
\hline & $\begin{array}{l}\text { Unité spatiale } \\
\text { élémentaire }\end{array}$ & Justification & Dimension de la fenêtre & Justification \\
\hline Lubéron & 2,25 hectares & Représentation des champs & $\begin{array}{l}163 \times 368 \text { pixels }= \\
54558 \text { hectares }\end{array}$ & $\begin{array}{l}\text { Trois géoterroirs avec prise en } \\
\text { compte de toutes les communes }\end{array}$ \\
\hline Larzac & 1 hectare & $\begin{array}{l}\text { Accrus de pins et taille des } \\
\text { champs }\end{array}$ & 6000 hectares & Ensemble des propriétés \\
\hline Pyrénées & 200 mètres carrés & $\begin{array}{l}\text { Conservation de limites des } \\
\text { petites parcelles de gestion } \\
\text { des exploitants }\end{array}$ & 15 kilomètres carrés & Commune étudiée de Villelongue \\
\hline Ferlo & 1 kilomètre carré & $\begin{array}{l}1 \text { kilomètre carré mais } \\
\text { association d'objets } \\
\text { vecteurs et raster }\end{array}$ & $100 \times 110 \mathrm{~km}$ & $\begin{array}{l}\text { Grande mobilité des éleveurs pour } \\
\text { l'accès à l'eau (fleuve, lac, forages) }\end{array}$ \\
\hline Pampa & 100 hectares & Taille des champs & 20000 kilomètres carrés & région homogène \\
\hline Amazonie & 4,5 hectares & $\begin{array}{l}\text { l'« Alqueire » est la base } \\
\text { d'échange }\end{array}$ & $\begin{array}{l}100 \text { hectares } \times N \\
(N=\text { nb de lots } \approx 100)\end{array}$ & $\begin{array}{l}1 \text { ou } 2 \text { "Traversões" } \\
\text { N spécifié par l'utilisateur }\end{array}$ \\
\hline
\end{tabular}


Tableau 3. Les échelles temporelles des modèles.

Table 3. Temporal scales of the models.

\begin{tabular}{|c|c|c|c|c|}
\hline & Pas de temps & Justification & $\begin{array}{l}\text { Durée de } \\
\text { simulation }\end{array}$ & Justification \\
\hline Lubéron & Mois & Calendrier pastoral & 10 ans & Changements de paysages à l'échelle 15 ans \\
\hline Larzac & Semaine & Calendrier de travail sur la semaine & 10 ans & Rotation forestière sur 70 ans \\
\hline Pyrénées & 15 jours & $\begin{array}{l}\text { Calendrier des opérations } \\
\text { techniques (fauche, pâturage) }\end{array}$ & 30 ans & Dynamique du frêne \\
\hline Sénégal & $\begin{array}{l}\text { Mensuel } \\
\text { agrégé en } \\
\text { cinq saisons }\end{array}$ & $\begin{array}{l}\text { Correspondant aux pratiques } \\
\text { traditionnelles }\end{array}$ & 20 ans & $\begin{array}{l}\text { Normales pluviométriques disponibles } \\
\text { et classes d'âge d'éleveurs }\end{array}$ \\
\hline Pampa & Année & Décisions sur la campagne agricole & $\begin{array}{l}10 \text { ans } \\
\text { ou plus }\end{array}$ & $\begin{array}{l}\text { Étudier de l'arrivée récente } \\
\text { des grandes entreprises capitalistiques }\end{array}$ \\
\hline Amazonie & Semestre & $\begin{array}{l}\text { Saison des pluies et saison sèche } \\
\text { liée à disponibilité en main-d'œuvre }\end{array}$ & $25-30$ ans & $\begin{array}{l}\text { Pas de dynamique de population } \\
\text { prévue dans le modèle }\end{array}$ \\
\hline
\end{tabular}

taille du plus petit échantillon représentatif (géoterroirs dans le Lubéron, territoire d'une commune dans les Pyrénées).

Si tous les modèles ont choisi de discrétiser le temps (aucune gestion événementielle n'a été retenue), les granularités temporelles s'avèrent très disparates. Leur choix est piloté par la capacité à représenter les processus naturels (par exemple, croissance fourragère) ou les activités (fauche, coupe d'arbres, vente de propriété, etc.). Quatre critères justifient ces choix :

- contraintes de disponibilité en temps de travail (Larzac, Amazonie) ;

- saisonnalité de la production pastorale (Lubéron, Ferlo) ;

- calendrier des opérations de gestion des prairies et des troupeaux (Pyrénées) ; - fréquence de décisions clés (réunion annuelle des conseils ruraux au Ferlo).

Ces contraintes se reflètent sur les choix des pas de temps qui opèrent à l'échelle de la semaine ou de l'année selon les modèles (tableau 3). Un pas annuel est souvent retenu pour les bilans techniques et économiques, et la mise à jour de dynamiques écologiques (transition du Land Cover) (figure 3).

La durée des simulations est associée à plusieurs critères :

- le délai nécessaire pour observer des changements dans la dynamique de végétation (accrus de pins et formation de forêts sur le Larzac, colonisation des prairies par le frêne dans les Pyrénées); - la garantie de validité de certaines hypothèses (non-disparition des actifs actuels dans les systèmes d'élevage du Lubéron) ;
- l'horizon temporel pour percevoir les effets d'une politique publique ou les changements climatiques (Ferlo).

\section{Discussion}

\section{Ontologie ou simulation ?}

Présenter aux autres chercheurs du projet Trans, la structure de ce métamodèle a permis de partager et d'échanger des points de vue sur les SEE. Certains ont soulevé des manques tels que l'absence de la filière ou du marché. Celui-ci a été rajouté pour fixer les prix des produits agricoles. Mais son fonctionnement ne dépend pas de celui des agents: les échelles des cas d'étude sont trop réduites pour influencer les prix qui sont généralement indexés sur les marchés internationaux. Dans plusieurs modèles, les prix sont des variables de forçage dont les valeurs ne dépendent pas des agents mais sont fournies de façon externe. L'intérêt est alors de tester les facultés d'adaptation des producteurs à ces perturbations.

La partie "Agent " et "Collectivité " a soulevé des débats. L'absence de réseaux sociaux contribuant à l'émergence de normes culturelles a été pointée. De même, l'absence des membres individualisés de la famille, qui empêche notamment de prendre en compte le rôle des femmes, a été perçue comme une lacune. Ces remarques ont conduit à des discussions sur l'utilité même de la modélisation.
Nous défendons l'idée que le but principal de la modélisation n'est pas de mimer la réalité mais d'essayer de la comprendre. Plutôt que de tendre vers un réalisme illusoire, il est préférable de rechercher les mécanismes élémentaires qui caractérisent le système et en dirigent les grandes tendances. Ainsi, sur un même terrain, divers modèles peuvent être proposés en fonction des questions posées. Si l'objectif avait été de montrer le poids du travail salarié hors des exploitations, nul doute que les modèles auraient distingué les membres de la famille et montré le rôle des femmes dans les SEE.

De plus, il est essentiel de saisir la différence entre un modèle de représentation et un modèle de simulation. En science de l'information, on nomme le premier une ontologie: un mode de représentation des connaissances dont les concepts sont organisés dans un graphe pour structurer la dimension sémantique du système et rendre visible les relations entre les entités. Son objectif n'est pas d'aboutir à un modèle de simulation (Bommel et Müller, 2007). Toutefois, la conception d'un simulateur nécessite souvent d'utiliser les outils des ontologies tels que les schémas UML. Mais la simulation n'est pas une simple représentation. En plongeant le modèle dans le temps, elle révèle les conséquences cumulatives des activités des entités et de leurs interactions. Au-delà de la structure du modèle, elle fait émerger des propriétés souvent insoupçonnées. Or, ajouter dans une simulation des entités de type norme culturelle ou politique publique implique d'être capable de préciser leurs caractéristiques structurelles mais aussi dynamiques. Il faut donc pouvoir spécifier leur comportement, leur cycle de vie 
(apparition, changements d'état, disparition) et la façon dont elles influencent les agents (et inversement). C'est une chose de reconnaitre l'existence de telles entités, c'en est une autre de simuler leur évolution. Nul doute qu'elles n'influent sur la dynamique globale, mais démontrer la façon dont elles procèdent paraît encore hors de portée. D'ailleurs, ce n'est pas l'objectif des modèles développés. La différence entre une ontologie et un modèle de simulation s'avère encore difficile à saisir et soulève des interrogations sur la manière de concevoir un SMA. Elle trouve sa place dans le débat entre les partisans de l'approche KISS (Keep It Simple Stupid) et ceux de l'approche KIDS (Keep It Descriptive Stupid) (Edmonds et Moss, 2004).

\section{Faible généricité des dynamiques des agents}

Si l'évolution du Land Cover est partagée, l'absence de consensus sur les dynamiques des activités des agents pose question, car cela diminue la généricité du métamodèle. En effet, les comportements sont trop dissemblables d'un modèle à l'autre et il n'a pas été possible de trouver une règle commune d'organisation des activités pastorales. Certains modèles décrivent finement la gestion du troupeau et des parcours, quand d'autres s'attachent davantage à la gestion patrimoniale ou de la main d'œuvre. Il est impossible d'harmoniser les pas de temps sans contraindre les comportements des agents.

La raison de cette incompatibilité réside dans la variété des situations étudiées et des objectifs assignés aux modèles. Elle provient aussi de la manière dont la modélisation a été menée et qui obéit plus ou moins fidèlement aux calendriers des activités. Bien que ces derniers aient été établis sur chaque terrain à partir d'entretiens avec les producteurs, leurs traductions en schémas d'activités divergent. Certains modèles cherchent à en reproduire fidèlement les étapes, quand d'autres tentent de s'en détacher. Les premiers visent à plus de précision et les seconds à plus d'abstraction. Ainsi, le respect rigoureux du calendrier entraîne une perte de généricité. À l'inverse, une montée en abstraction tente de dépasser le déroulement figé des activités saisonnières pour essayer de saisir les raisons de la mise en place de tels calendriers. Elle cherche à dépasser la description contextuelle pour gagner en généricité.
Mise à part la divergence des objectifs des modèles, ces deux façons (descriptive ou adaptative) de représenter les comportements des acteurs génèrent des types de modèles difficilement comparables. Ceci explique en partie pourquoi ce métamodèle n'a pas encore atteint le stade de modèle générique.

\section{Conclusion}

Étudier les différences entre les systèmes d'élevage extensif du projet Trans est une façon pertinente d'acquérir des connaissances sur les SEE, mais nous avons préféré rechercher les points communs à l'ensemble des SMA développés sur les différents terrains. Ainsi, la conception d'un métamodèle a conduit à uniformiser les représentations des divers SEE pour mieux en comprendre la structure générale et les fonctionnements. Elle a permis des discussions enrichissantes et a demandé de la part des différentes équipes un retour critique sur leur propre modèle. Ce métamodèle est donc le fruit d'âpres négociations qui ont débouché sur un outil de dialogue favorisant le partage des connaissances et l'interdisciplinarité.

Mais il s'agit encore d'un premier essai, car il reste du travail de comparaison et de conception à mener pour identifier les invariants des SEE afin d'en saisir les éléments essentiels permettant d'expliquer la diversité des dynamiques. Il est pourtant vain d'espérer trouver un tel modèle générique capable de décrire l'ensemble des SEE malgré la diversité des contextes. Sans qu'on puisse espérer y parvenir, il s'avère néanmoins nécessaire de délaisser les approches purement descriptives au profit de modèles plus adaptatifs.

\section{Références}

Bah A, Touré I, Fourage C, Diop Gaye I, Leclerc G, Soumaré A. Un modèle multi-agents pour étudier les politiques d'affectation des terres et leurs impacts sur les dynamiques pastorales et territoriales au Ferlo (Sénégal). Cahiers Agric 2010 ; 19 : epub. DOI : 10.1684/agr.2010.0383.

Bommel P. Définition d'un cadre méthodologique pour la conception de modèles multiagents adaptée a la gestion des ressources renouvelables. Thèse de I'université de Montpellier II, sciences et techniques du Languedoc, 2009.
Bommel P, Müller JP. An introduction to UML for modeling in the human and social sciences. In : Amblard F, Phan D. eds. Introductory book on MAS modeling and simulation in Human and Social Sciences. Oxford: The Bardwell Press, 2007.

Bommel B, Bonaudo T, Barbosa T, Bastos da Veiga J Vieira Pak $M$, Tourrand JF La relation complexe entre I'élevage et la forêt en Amazonie brésilienne : une approche par la modélisation multi-agents. Cah Agric 2010; 19 : epub. DOI : 10.1684/agr.2010.0384

Bousquet F, Bakam I, Proton H, Le Page C. Cormas: Common-Pool Resources and Multiagent System. Lectures Notes in Artificial Intelligence $1998 ; 1416$ : 826-38.

ComMod. collectif. La modélisation comme outil d'accompagnement. Nature Sciences et Sociétés 2005; 13 : 165-9.

Edmonds B, Moss S. From KISS to KIDS - an 'anti-simplistic' modelling approach. In : Davidsson P, Logan B, Takadama K, eds. Multi Agent Based Simulation. Lecture Notes in Artificial Intelligence. Heidelberg : Springer, 2004

Etienne M, Dobremez L, Guérin G, Rapey H, Simon C. Une modélisation d'accompagnement pour la gestion combinée des systèmes d'élevage et des milieux boisés sur le Causse du Larzac. Cah Agric 2010; 19: epub. DOI: 10.1684/agr.2010.0374

Ferber J, Gutknecht O. A meta-model for the analysis and design of organizations in multiagent systems. In : Third International Conference on Multi-Agent Systems (ICMAS '98) Proceedings. IEEE Computer Society, 1998.128-135.

Monteil C. Simon C, Ladet S, Sheeren D, Etienne M, Gibon A. Participatory modelling of social and ecological dynamics in mountain landscapes subjected to spontaneous ash reforestation. In : Paegelow M, Camacho-OImedo $\mathrm{MT}$, eds. Modelling Environmental Dynamics. Advances in Geomatic Solutions. Heidelberg : Springer, 2008.

Goldspink C. Modelling social systems as complex: Towards a social simulation meta-model. Journal of Artificial Societies and Social Simulation 2000 ; 3 : epub. http://jasss.soc.surrey. ac.uk/3/2/1.html

Grimm V, Railsback SF. Individual-based Modeling And Ecology. Princeton : Princeton University Press, 2005.

Lasseur J, Bataille JF, Beylier B, Etienne M, Legeard JP, Luccioni J. Modélisation des relations entre dynamiques des territoires et des systèmes d'élevage dans le massif du Lubéron. Cah Agric 2010 ; 19 : epub. DOI : 10.1684/ agr.2010.0376.

Morales GH, Arbeletche P, Bommel P, et al. Modéliser le changement dans la gestion des terres de parcours en Uruguay. Cah Agric 2010 ; 19 : epub. DOI : 10.1684/agr.2010.0385

OMG. OMG Specification of Unified Modeling Language. Version 1.5, document Number: formal/03-03-01. Object Management Group, 2003 . http://www.omg.org/cgi-bin/doc?formal/ 03-03-01.

Orléan A. What is a Collective Belief. In : Bourgine $\mathrm{P}, \mathrm{Nadal} \mathrm{JP}$, eds. Cognitive Economics, An Interdisciplinary Approach. Heidelberg : Springer, 2004. 